"chan" — 2008/7/31 - 11:51 — page $63-\# 1$

\title{
Different approaches of interplay between experimentation and theoretical consideration in dynamic geometry exploration: An example from exploring Simson line
}

\author{
YiP-Cheung Chan
}

\begin{abstract}
Dynamic geometry environment (DGE) is a powerful tool for exploration and discovering geometric properties because it allows users to (virtually) manipulate geometric objects. There are two possible components in the process of exploration in DGE, viz. experimentation and theoretical consideration. In most cases, there is interplay between these two components. Different people may use DGE differently. Depending on the specific mathematical tasks and the background of individual users, some approaches of interplay are more experimental whereas some other approaches of interplay are more theoretical. In this paper, different approaches of exploring a geometric task using Sketchpad (a DGE) by three individual participants will be discussed. They represent three different approaches of interplay between experimentation and theoretical consideration. An understanding of these approaches may contribute to an understanding on the mechanism of exploration in DGE.
\end{abstract}

Key words and phrases: dynamic geometry environment, computer-aided exploration, experimentation, theoretical consideration.

ZDM Subject Classification: U70.

\section{Introduction}

Dynamic geometry environment (DGE) is a powerful tool for exploration and discovering geometric properties because it allows users to (virtually) manipulate geometric objects. As Goldenberg and Cuoco points out, "dynamic geometry 
"chan" - 2008/7/31 — 11:51 — page $64-\# 2$

environment allows users, after a construction is made, to move certain elements of a drawing freely and to observe other elements respond dynamically to the altered conditions" ([3, p. 351]). However, it seems that only a few studies have examined the mechanism of exploration in DGE in detail. In contrast, extensive research has been done on investigation of the exploration process in computer algebra system (CAS). Instrumentation theory $([13])$ is one of the theoretical frameworks usually adopted in these research studies (see e.g. [1], [4], [5], [11]).

The central idea of instrumentation theory is the double interpretation of a technical device, say DGE. It is an artifact, which means an object designed for some purposes. However, the way of using an artifact is situational in the sense that a particular artifact may be used very differently in different situations and by different users (or subjects, in the terminology of instrumentation theory). An artifact associates with its utilization scheme in a particular situation by a particular user is called an instrument. Drijvers and Gravemeijer define utilization scheme as "a stable mental organization which includes both technical skills and supporting concepts for a way of using the artifact for a given class of tasks" ([2, p. 167]). The process of developing an artifact into an instrument, called as instrumental genesis by [13], is a complicated process and involves two interrelated components. On the one hand, it "directed toward the artifact" and is called instrumentalization $([12$, p. 145]). On the other hand, it "directed toward the subject" and is called instrumentation ([12, p. 145]). In other words, it is a two-way interaction between the subject and the artifact.

In [7], [8], [9], variational dragging scheme is proposed as a perspective to analysis instrumental genesis in DGE. In this paper, another perspective to understand instrumental genesis in DGE will be discussed.

The exploration in DGE usually involves experimentation and theoretical consideration. In the light of instrumentation theory, it seems that there is some kind of interaction between these two components. On the one hand, the visual feedback provided by DGE experimentation gives a ground for the user's developments of theoretical understandings of the task and technical skills of using the software. On the other hand, the method of conducting experiments in DGE is influenced by the user's theoretical consideration of the task and his/her competence of using the software. Thus, an understanding of the interplay between experimentation and theoretical consideration may contribute to an understanding on the mechanism of exploration in DGE (in particular, on instrumental genesis in DGE). 


$$
\text { "chan" — 2008/7/31 — 11:51 — page } 65-\# 3
$$

In this paper, some possible approaches of interplay between experimentation and theoretical consideration in exploration in DGE will be described. These approaches will be illustrated by observations from an empirical study. At the end of this paper, some possible directions for further research will be proposed.

\section{An empirical study}

In this empirical study, three participants ${ }^{1}$ who have strong mathematics background worked individually to explore a geometrical task in Sketchpad (a DGE). The following is the instruction of the task:

Use Sketchpad to construct the following geometrical configuration: $A B C$ is a triangle on a plane. $P$ is an arbitrary point on that plane. Let $X$, $Y, Z$ be the feet of the perpendicular lines drawn from $P$ to the sides $A B, B C$ and $A C$ respectively. (Sides $A B, B C$ and $A C$ can be extended if necessary.) Use Sketchpad, find all possible positions of $P$ such that $X, Y, Z$ are collinear (i.e. $X, Y, Z$ lie on the same straight line).

This task is designed according to Simson's theorem (see e.g. [10, pp. 61-68]). The theorem says:

Consider triangle $A B C$. Let $P$ be a point and $X, Y, Z$ be the feet of perpendicular lines drawn from $P$ to the (extended) sides $A B, B C$ and $A C$ respectively. The locus of $P$ such that $X, Y, Z$ are collinear is the circumscribed circle of triangle $A B C$. (The line joining $X, Y, Z$ is called Simson line.)

In other words, the expected answer of this task is that the locus of $P$ is the (unique) circle containing the three vertices $A, B$ and $C$.

Figure 1 is a typical dynamic figure constructed by the participants. Vertices $A, B, C$ and point $P$ are free points. $X, Y, Z$ are intersection points and hence are fixed.

As we may expect, after constructing the figure, the participants dragged $P$ in a wandering fashion aimed at making $X, Y, Z$ visually collinear. This action gave them some feelings to the task and suggested them a possible direction of exploration.

${ }^{1}$ All names reported in this paper are pseudonyms. 


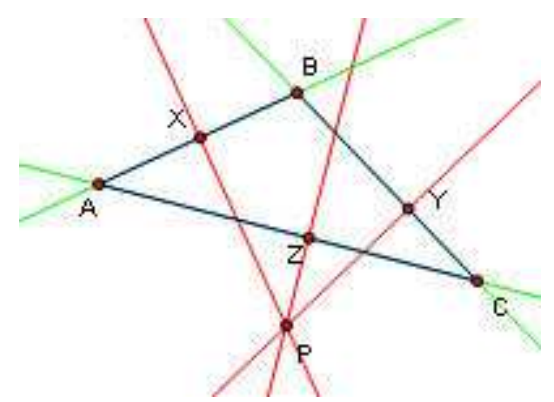

Figure 1. Typical dynamic figure constructed by the participants.

In the following discussion, I will describe how three participants (individually) working on the task after dragging $P$ for a while. They represent different approaches of explorations in DGE.

For convenience, the following abbreviations will be used.

- Condition $\mathcal{C}$ is satisfied if points $X, Y, Z$ are collinear

- A position of $P$ is said to be suitable if it leads condition $\mathcal{C}$ to be satisfied.

\subsection{Case 1 (Tracy)}

Tracy is a female mathematics teacher in a secondary school. She obtained a Bachelor degree in mathematics and was studying part time Master of Science in mathematics when this research project was conducted. She knew basic commands of Sketchpad but seldom used this software in her teaching.

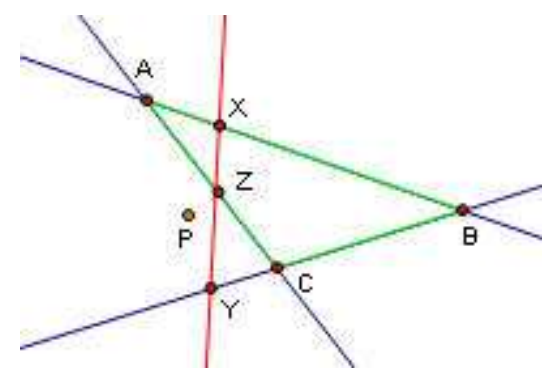

Figure 2. Line $X Z$ was constructed as a visual guide for locating suitable positions of $P$.

After trying to locate suitable positions of $P$ randomly for a while, she realized that it was not accurate enough to determine whether condition $\mathcal{C}$ was satisfied 


$$
\text { "chan" — 2008/7/31 - 11:51 — page } 67-\# 5
$$

only by naked eyes. In order to increase the reliability of visual observation, she constructed a line joining points $X$ and $Z$. $P$ was dragged keeping $Y$ as a point on the line (at least visually). The line served as a "visual guide" for locating suitable positions of $P$. (See Figure 2.) This was a guided drag/drag-tofit strategy. Specific examples of suitable positions of $P$ were located by trial-anderror experimental method. Definition of collinearity (theoretical consideration) suggested an auxiliary tool for the experimentation.

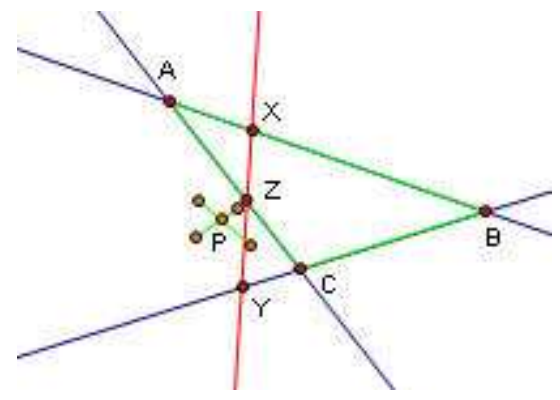

Figure 3. A "cross" was made to "mark" the found position of $P$.

In order to guess the locus, Tracy needed to record those suitable positions of $P$. Initially, she used trace function but it was not satisfactory because all positions of $P$, no matter condition $\mathcal{C}$ was satisfied or not, were marked by trace function. In order to overcome this difficulty, she invented her own method. A "cross" was made by two line segments to "mark" the position (Figure 3). (Then, an intersection point was constructed and the two line segments were hidden.) This self-developed utilization technique is very similar to the method of marking positions on paper. It seems to be a transfer of skills from paper-andpencil environment to DGE. The user's experience of working in paper-and-pencil environment influenced her development of utilization technique of the software.

After repeating this method several times, some suitable positions of $P$ have been found and marked down. According to the figure shown on screen (Figure 4), the participant guessed that the locus of suitable positions of $P$ is a circle but she was not sure what is that circle.

Now, she dragged $P$ to other positions on the plane such as the interior of triangle $A B C$. Accidentally, she realized that condition $\mathcal{C}$ was satisfied when $P$ was positioned at vertex $C$. As we may expect, she dragged $P$ to the other two vertices $A$ and $B$ and realized that condition $\mathcal{C}$ was also satisfied. That means, $A, B, C$ were points of the required locus. Based on her knowledge about circle 


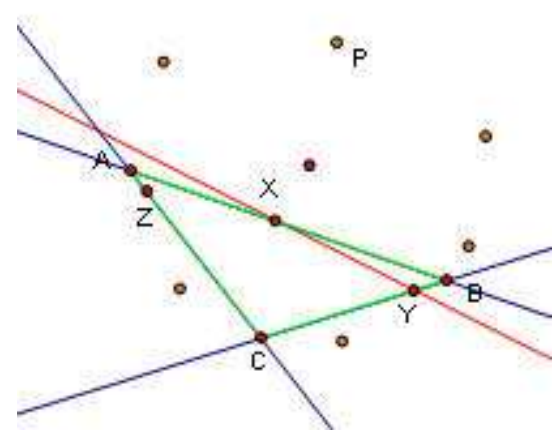

Figure 4. The marked points shown on screen suggested that the locus is a circle.

(three points defines a unique circle), she guessed that the required locus is the circumscribed circle of triangle $A B C$. Her mathematical knowledge contributed to the interpretation of findings of the experiment and enriching the experimentation results.

Based on her mathematical knowledge (the center of the circumscribed circle is the intersection point of perpendicular bisectors), Tracy constructed the circumscribed circle of triangle $A B C$ in Sketchpad, and then observed that all previously marked points lied on that circle (at least visually). This further enhanced her confidence that she had made a correct guess.

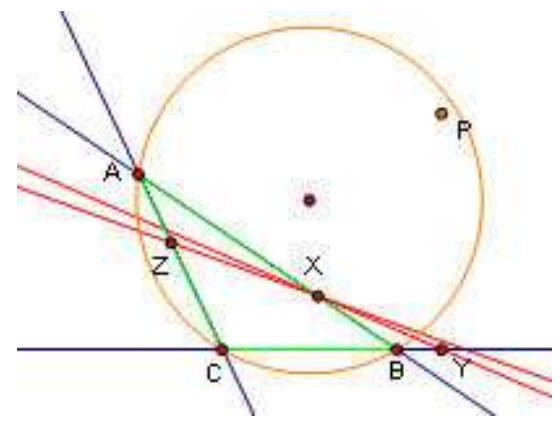

Figure 5. Line $X Z$ and line $X Y$ were used for ensuring that $X, Y, Z$ are collinear.

In order to further verify the conjecture, $P$ was merged to the circumscribed circle. The geometric configuration was changed: $P$ was no longer a free point but bounded on the circumscribed circle. Then, animation function was used to 


$$
\text { "chan" - 2008/7/31 - 11:51 — page } 69-\# 7
$$

let $P$ moved along the circle automatically, and thus $X, Y, Z$ were always lying on a line. This provided a dynamic demonstration of the sufficient condition for condition $\mathcal{C}$, i.e. if $P$ is a point on the circumscribed circle, then $X, Y, Z$ are collinear.

After playing with the dynamic figure for a while, Tracy doubted on the accurateness of visual observation by her naked eyes. In order to further ensure $X, Y, Z$ were really collinear, she constructed one more line joining $X$ and $Y$ (Figure 5). Then, $P$ was merged on the circumscribed circle. She observed that line $X Z$ was overlapped with line $X Y$. This further confirmed that the required locus has been found. It seems that the mathematical property "three points are collinear if and only if the lines joining different pairs of these points have same slope" was evoked (theoretical consideration) to develop a method for further verification of the conjecture (experimentation).

\subsection{Case 2 (Kelvin)}

Kelvin is a male mathematics teacher in a secondary school. He obtained a Bachelor degree and a Master degree in mathematics and was studying part time Master of Education when this research project was conducted. He was familiar with Sketchpad and sometimes used this software as an auxiliary tool for his teaching.

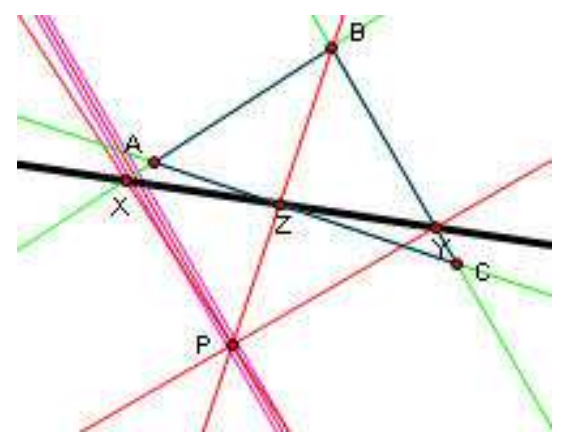

Figure 6. Checking whether $X P$ is parallel to $B C$.

Similar to what Tracy did, after trying to locate suitable positions of $P$ by trial-and-error for a while, Kelvin constructed a line joining $X$ and $Y$ to guide a systemic search of suitable positions of $P$. However, he did not try to "mark" the found suitable positions of $P$ as Tracy did because he did not know how to mark 
these positions. He realized that he could not put a "nail" on the suitable position and trace function would mark both suitable positions and unsuitable positions in wandering dragging. Instead, he tried to guess geometric relationships between a suitable point $P$ and other points on the figure (i.e. $X, Y, Z, A, B, C$ ) based on his visual intuition.

The first guess was that $X P$ is parallel to $B C$. He checked it by constructing a line parallel to $B C$ (Figure 6 ). The parallel line did not overlap with line $X P$. Hence, the guess was rejected.

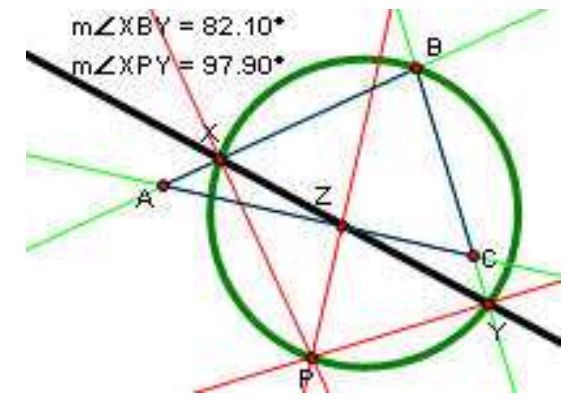

(a) $X P Y B$ is concylic when $X, Y, Z$ are collinear.

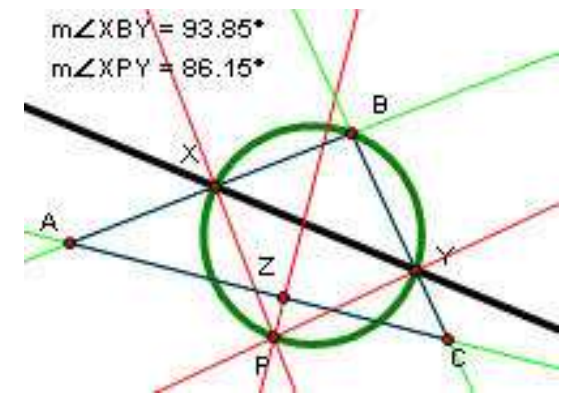

(b) $X P Y B$ is concylic when $X, Y, Z$ are non-collinear.

Figure 7

The second guess was that $X P Y B$ is a cyclic quadrilateral. He constructed a circumscribed circle of triangle $X P Y$. Then, he randomly dragged $P$ and realized that $B$ always lies on that circle (Figure $7(\mathrm{a})$ ). However, after dragging for a while, he realized that this happened even if condition $\mathcal{C}$ was not satisfied (Figure $7(\mathrm{~b})$ ). This implies that the property " $X P Y B$ is a cyclic quadrilateral" is not a condition for $X, Y, Z$ to be collinear. Sketchpad experimentation of contrasting different cases led to refutation of the guess.

It seems that Sketchpad visual observation did not help him much. Instead of carrying on the experimental approach, Kelvin worked on paper-and-pencil environment. He used traditional coordinate geometry method to find the equation of locus of $P$. Figure 8 shows the beginning part of his paperwork. Pure theoretical computation approach was adopted.

After tedious symbolic computations, he found that the equation of locus of $P$ is $x^{2}+y^{2}-x+\left(\frac{u+v-u^{2}-v^{2}}{v}\right) y=0$. Based on this equation, he knew that the locus is a circle with $x$-coordinate of center $=\frac{1}{2}$. However, he did not satisfy to this answer because he wanted to find a geometric interpretation of this equation. 


$$
\text { "chan" - 2008/7/31 - 11:51 - page } 71-\# 9
$$

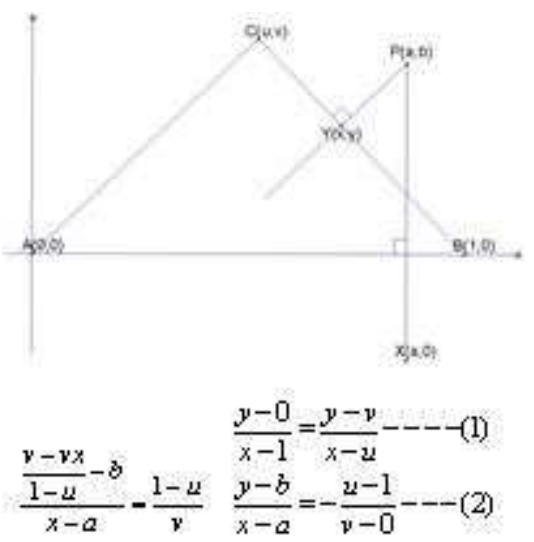

Figure 8. Beginning part of Kelvin's paperwork.

He tried to construct the locus by Sketchpad according to his earlier computations. As points $A$ and $B$ were defined as $(0,0)$ and $(1,0)$ respectively, $x$-coordinate of center $=\frac{1}{2}$ implies that the center lies on the perpendicular bisector of $A B$. Then, he realized that the center should also lie on the perpendicular bisector of the other two sides of the triangle. Thus, the center has to be the intersection point of perpendicular bisectors of the sides of triangle $A B C$.

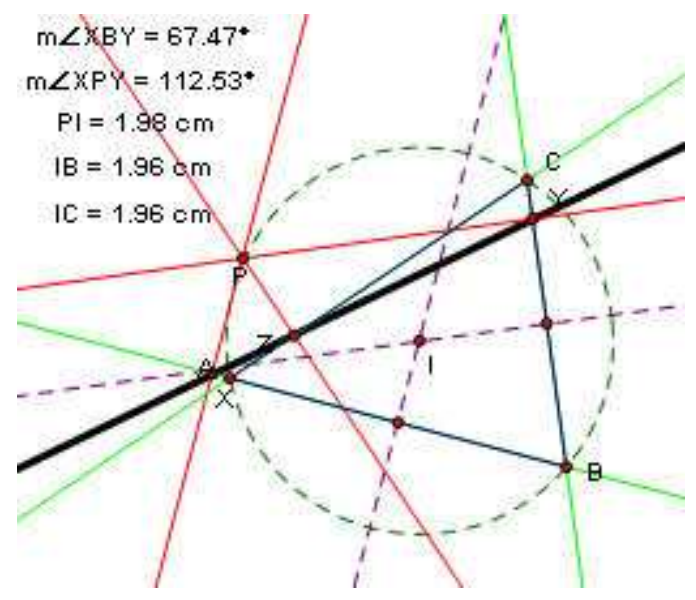

Figure 9. Finding the geometric meaning of the radius by Sketchpad experiment. 
He further tried to find the geometric meaning of the radius but theoretical considerations could not help him much. Thus, he switched back to Sketchpad experimentation. First, he measured the lengths of $P I, I B$ and $I C$ where point $I$ is the center. Then, $P$ was dragged such that condition $\mathcal{C}$ was satisfied by drag-tofit strategy. He realized that the three lengths were more-or-less equal implying that they are indeed the radii of the circle. In other words, locus of $P$ should be the circumscribed circle of triangle $A B C$. This was verified by constructing a circle with $I$ as the center and $I B$ as the radius (Figure 9).

\subsection{Case 3 (Samuel)}

Samuel is a male university mathematics instructor. He obtained a $\mathrm{PhD}$ degree in mathematics. He did not know Sketchpad before this research project was conducted. He was a little bit reluctance to use the software and tried his best to use the software according to the logical sequence of Euclid's Element. (For example, he did not use some built-in Sketchpad functions such as measure, trace and merge in the exploration process because he thought that these functions are incompatible to traditional Euclid geometry.)

After trying to locate suitable positions of $P$ by trial-and-error for a while, he focused his attention on finding which regions are impossible to yield condition $\mathcal{C}$. His initial speculation was that $P$ should not be positioned in the interior of triangle $A B C$. In order to confirm this speculation, he changed the shape of triangle $A B C$ by dragging vertex $A$ randomly. Then, the boundaries of triangle $A B C$ and the three vertices $A, B, C$ were explored. The observations were formulated as conjectures by formal mathematics languages.

Denote by $\Delta$ the triangle $\triangle A B C, \Delta^{o}$ the interior of $\triangle A B C, \partial \Delta$ the

boundary of $\triangle A B C, V(\Delta)=\{A, B, C\}$.

Conjecture (1): If $P \in \Delta^{o}$ then $X, Y, Z$ cannot be collinear.

Conjecture (2): Suppose $P \in \Delta$. Then $X, Y, Z$ are collinear iff $P \in V(\Delta)$.

In this case, two amongst $X, Y, Z$ coincide with $P$.

$(2) \Rightarrow(1)$

Now, $P$ was dragged randomly outside triangle $A B C$ and different regions were classified. They were described by formal mathematics languages as shown in Figure 10.

Theoretical consideration (classification of regions) led the direction of experimental exploration. First, he explored type (I) region by randomly dragging $P$ within $\Pi^{\circ}$ (a region of type (I)). He tried to check whether it was possible to 


$$
\text { "chan" — 2008/7/31 — 11:51 — page } 73-\# 11
$$

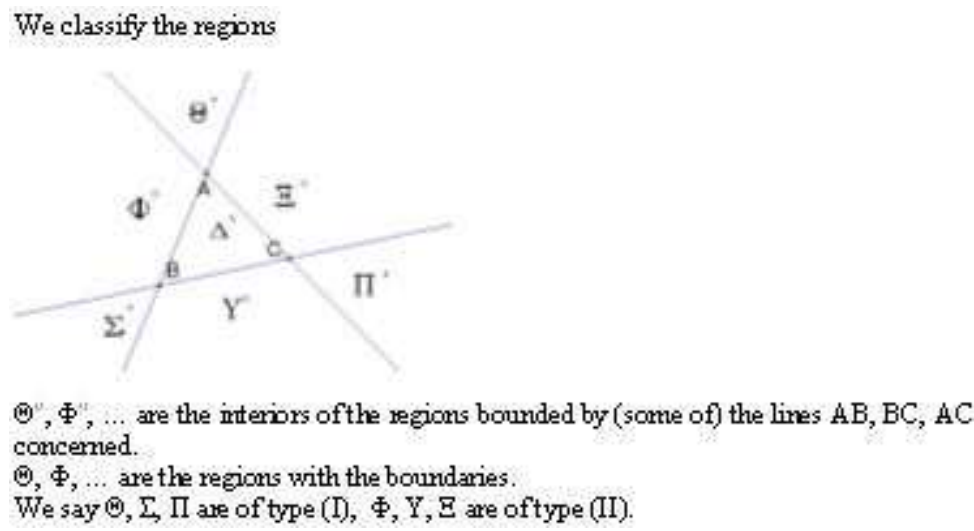

Figure 10. Classification of regions.

produce the required condition $\mathcal{C}$ when $P$ was located within regions of type (I). Similarly, he explored type (II) region by randomly dragging $P$ within $\Upsilon^{o}$ and $\Xi^{o}$ (regions of type (II)). The observations were formulated as a conjecture by formal mathematics languages.

Conjecture (3): Suppose $P \in \mathbb{R}^{2} \backslash \Delta$. Then $X, Y, Z$ are collinear iff $P$ is in the interior of one of the type (II) regions, i.e. $\Phi^{o}, \Upsilon^{o}, \Xi^{o}$.

Corollary to conjecture (3): Suppose $P \in k \cup l \cup m$ where $k, l, m$ are the (infinite) lines containing $\{A, B\},\{B, C\},\{A, C\}$ respectively. If $X$, $Y, Z$ are collinear then $P \in V(\Delta)$ and two of $X, Y, Z$ must coincide with $P$.

Now, he tried to narrow down the coverage of exploration to type (II) regions only. His prior knowledge (theoretical consideration) directed further experimentation and led to deeper exploration of type (II) regions. He dragged $P$ within regions of type (II) and realized some patterns: if $P$ is far away from the triangle, it seems impossible for condition $\mathcal{C}$ to be satisfied (Figure 11(a)); if $P$ is near to the triangle, it seems to have more chances to satisfy condition $\mathcal{C}$ (Figure 11(b)).

By contrasting on different cases, Samuel guessed that $P$ should be a point lying on the circle connecting the three vertices $A, B, C$ in order to satisfy condition $\mathcal{C}$. His intuition and mathematical knowledge of geometric figures provided insights to interpret the experimental observations. (Probably, he was implicitly thinking about the relative positions of points of a circumscribed circle to its 


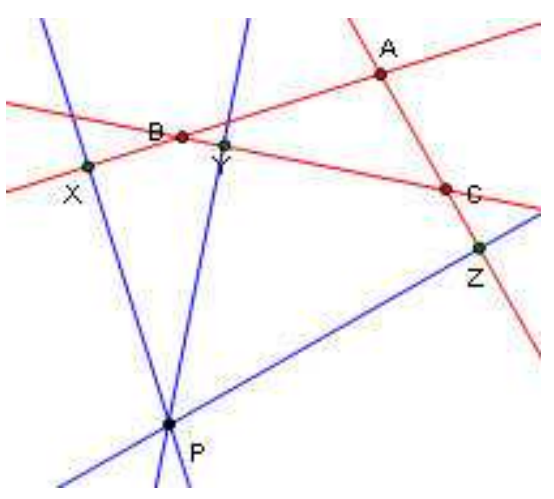

(a) Dragging $P$ far away from the triangle.

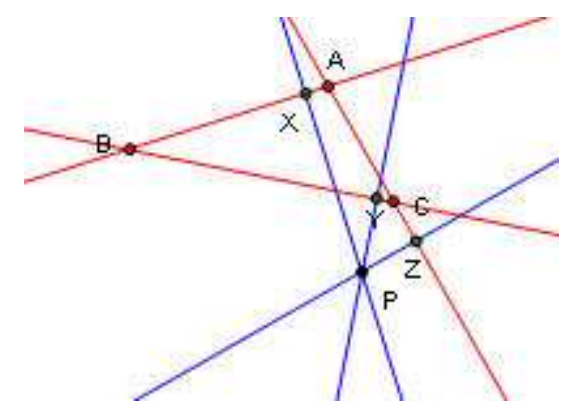

(b) Dragging $P$ near the triangle.

Figure 11

based triangle.) This led to a conjecture that was beyond observation, viz. the locus is the circumscribed circle.

Now, he constructed the circumscribed circle of triangle $A B C$ by Sketchpad. By fitting $P$ along the circle as close as possible (drag-to-fit strategy), a dynamic demonstration of the conjecture was exhibited. The conjecture was formulated by formal mathematics languages as below.

Conjecture (4): $X, Y, Z$ are collinear iff $P \in \Gamma$ where $\Gamma$ is the circle containing $A, B, C$.

Conjecture (4) renders everything prior to it redundant.

Further experiment was conducted for verifying the necessary part of the conjecture. The design of experiment was theoretical-based.

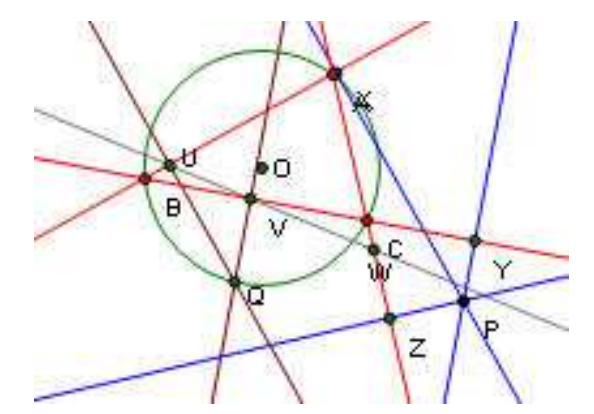

Figure 12. Further experiment for verifying the necessary part of the conjecture. 


$$
\text { "chan" — 2008/7/31 — 11:51 — page } 75-\# 13
$$

A point $Q$ was constructed on the circumscribed circle. Then, points $U$ and $V$ perpendicular to the extended lines $A B$ and $B C$ respectively were constructed. Finally, the intersection point $W$ of line $U V$ and line $A C$ was constructed. (See Figure 12.) Samuel recalled that $Q W$ is perpendicular to $A C$ if and only if $A C$ is a tangent line to the circle with $Q$ as its center and $Q W$ as its radius.

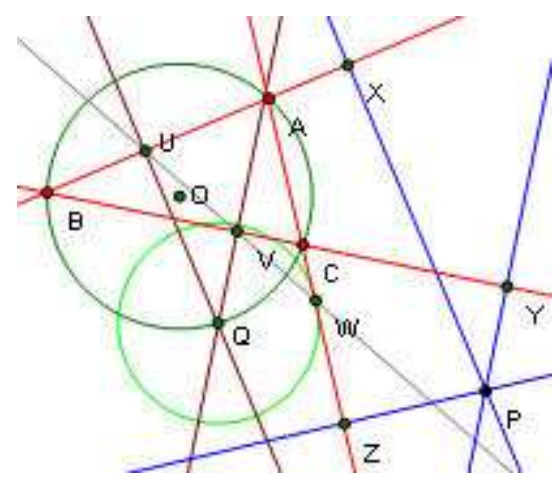

Figure 13. Another circle is constructed to verify that $Q W$ is perpendicular to $A C$.

Such a circle was constructed (Figure 13). He realized that $A C$ was always a tangent line to that circle when $Q$ was dragged along the circumscribed circle.

Geometric theory (Samuel's prior mathematical knowledge) guided the design of the experiment. It was a theoretical-based checking method. Now, he was confident that a correct conjecture has been made and tried to prove it by the notions of vectors. After attempting to prove the conjecture in paper-and-pencil environment for a while, he gave up.

\section{Discussion}

In the process of exploration in DGE, interplay between theoretical consideration and experimentation usually occurs. In the three cases described above, different approaches of interplay were observed. In the following sections, we will analysis these approaches in details. 


$$
\text { "chan" — 2008/7/31 - 11:51 — page } 76 \text { — \#14 }
$$

Yip-Cheung Chan

\subsection{Approach of interplay in Tracy's exploration}

Throughout the exploration, Tracy spent most of the time on experimentation in Sketchpad. Some critical features of her exploration are observed. First, she failed to use trace function to mark the found suitable positions of $P$. Then, she invented her own method to overcome this difficulty and continued the experiment. Second, in the process of checking whether $P$ could be put inside triangle $A B C$ by wandering dragging, she "accidentally" positioned $P$ at vertex $C$. This informed her which circle is the locus of $P$. It has demonstrated the power of DGE experiment. Insight could be evoked even in the case of seems-to-be un-strategic randomly dragging. Third, she was unsatisfied to the visual determination of condition $\mathcal{C}$. Thus, she "invented" her own method, viz. constructing two lines $X Z$ and $X Y$ and then checked whether they were overlapped. Although Tracy relied heavily on Sketchpad experimentation in her exploration, she took it with special care. She made effort to make the visual feedbacks as reliable as possible. As a consequence, some utilization techniques were developed.

Let us now summarize the approach of interplay between experimentation and theoretical consideration in Tracy's exploration. The exploration process depended heavily on experimentation with Sketchpad. Conclusions were made mainly based on visual observation of Sketchpad. However, she did not completely trust the observation of her naked eyes. She used her mathematical knowledge to develop techniques to increase the reliability of observations. In other words, experimentation took a prominent role throughout the exploration process in the sense that experimentation and Sketchpad visual observation directed the exploration process. Mathematical knowledge took the supporting role in the sense that it was used mainly for developing utilization techniques to actualize the experiments.

\subsection{Approach of interplay in Kelvin's exploration}

In the beginning of Kelvin's exploration, experimentation seems unable to help him. He did not try to mark down those suitable positions of $P$ because he thought that trace function could not help him. Instead of trying to overcome the difficulty as what Tracy did, he switched the path of exploration completely, viz. using coordinate geometry to find the equation of locus.

Although he successfully found the equation of locus which was indeed a rigorous mathematical proof, he did not satisfy to that answer and insisted to 


$$
\text { "chan" — 2008/7/31 — 11:51 — page } 77-\# 15
$$

find a geometric interpretation. This led him to go back to Sketchpad exploration. Sketchpad experimentation gave him geometric insight and he satisfied to this geometric answer. The whole process is very interesting. A (rigorous) mathematical proof did not satisfy to Kelvin but a Sketchpad experiment led to mathematical understanding instead. It seems that he satisfied to the finding based on Sketchpad exploration much more than that by coordinate geometry computations. This inspires us to think about some fundamental questions:

- What is proof? What is its role in mathematical discovery?

- What does coordinate geometry meant to Kelvin (or perhaps, more generally, to some mathematics teachers)? Is it really a mathematical knowledge (theoretical consideration) or is it just a "tool" (a set of algorithms) for solving some stereotyped mathematical problems?

Let us now summarize the approach of interplay between experimentation and theoretical consideration in Kelvin's exploration. During the exploration process, experimentation and theoretical consideration (mathematical knowledge) were partners (complemented to each other). Both experimentation and theoretical computation played crucial roles in the exploration process. They "worked together" to give a complete answer of the question.

\subsection{Approach of interplay in Samuel's exploration}

Although Samuel did experiment in the process of exploration, the experimentation was theoretical-led. First, the design of experiment was guided by a theoretical framework, viz. classification of regions. He tried to identify which regions are possible for condition $\mathcal{C}$ to be satisfied and which regions are impossible. The process of identification does not merely based on experimentation. Before checking different regions by randomly dragging, Samuel classified the six exterior regions into two types. He explained that type (I) region is bounded by two (extended) sides whereas type (II) region is bounded by three (extended) sides. Furthermore, he formulated the observations into conjectures by using abstract mathematical languages. It seems that he tried effort to connect the finding of experimentation to theoretical consideration. The intention of making concrete observations abstract seems related to his education backgrounds and working experiences.

Theory not only served as guidance for designing experiments but also for interpretation of experimental observations. In the process of further exploration of type (II) regions, he contrasted those positions that are near to the vertex 


$$
\text { "chan" — 2008/7/31 — 11:51 — page } 78-\# 16
$$

with those positions that are far apart. His intuition and knowledge of geometric figures led him to guess that the locus is a circle connecting the three vertices. His geometric insight plays a prominent role in the establishment of conjectures that are beyond experimental observations.

In order to verify the conjecture, Samuel developed another Sketchpad experiment. The experiment is theoretical-based. The aim of the experiment was to check the perpendicularity of $Q W$ and $A C$. Instead of using the built-in perpendicular command which Tracy and Kelvin might adopt, Samuel used the tangent property of a circle. This theoretical approach of designing experiment seems related to his perception of viewing Sketchpad as visual demonstrations of Euclid's geometry.

Let us now summarize the approach of interplay between experimentation and theoretical consideration in Samuel's exploration. Theoretical consideration (mathematical knowledge) played a prominent role throughout the exploration process. Experimentation plays a supporting role in the sense that it actualized the intentions produced by theoretical considerations such as testing suitable positions of $P$ and checking conjectures. Mathematical theory directed the goals and designs of experiments as well as the interpretations of experimental observations. Moreover, Samuel's perception of Sketchpad (in broad sense, it is another kind of theoretical consideration) also influenced how the experiments was conducted.

\subsection{Comparisons of different approaches}

In the above discussion, three different approaches of interplay between experimentation and theoretical consideration by different people working on same exploration task have been mentioned. These approaches can be summarized as below.

(1) Experimentation-driven approach (e.g. Tracy's exploration):

Experimentation plays a prominent role in the process of exploration. Conclusions are made based on experimental observations. Theory is used for development of methods to actualize the intended experiments. As attention is put on experimentation, the problem-solver may put his/her major effort on development of utilization schemes of using the software to do exploration.

(2) Experimental-theoretical-partnership approach (e.g. Kelvin's exploration): Experimentation and theoretical consideration complement to each other in the process of exploration. Both components play significant roles in reaching the answers of the task. The problem-solver swaps the attention between 


$$
\text { "chan" — 2008/7/31 - 11:51 — page } 79-\# 17
$$

experimentation and theoretical consideration depending on the progress of exploration.

(3) Theory-driven approach (e.g. Samuel's exploration):

Theoretical consideration plays a prominent role in the process of exploration. It directs the design of experiments and the interpretation of findings. Consequently, mathematical properties beyond experimental observations may be discovered. Experimentation actualizes theoretical considerations. As attention is put on theoretical consideration, the problem-solver may put his/her major effort on mathematical interpretation of experimental feedbacks and its relation to the given task. He/she may also formulate the observations into mathematical statements by abstract languages.

\subsection{Implication to future trends in interactive software}

Different people perceived and used Sketchpad differently. When interpreting the user's exploration process, it seems that we should not regard Sketchpad (or other computer software) as software that is the same for all problem-solvers but instead software of each individual. That means: Tracy's Sketchpad, Kelvin's Sketchpad and Samuel's Sketchpad are indeed different Sketchpad because the users perceived Sketchpad differently. This observation echoes to the finding of [6] (in the case of Cabri). A central idea of instrumentation theory is the development of utilization scheme. This study may contribute to an understanding on how to develop utilization schemes in DGE.

This study suggests an implication to future trends in interactive software. As different people use interactive software differently, flexibility for catering individual differences should be one of the major concerns in future development of interactive software. For example, both Tracy and Kelvin realized that trace function could not be used directly for marking found positions ("put a nail on the position"). However, their reactions were totally different so that two very different approaches of exploration have been led. What lessons about development of interactive software have we learnt?

\section{Concluding remarks}

In this paper, three approaches of interplay between experimentation and theoretical considerations in the process of exploration in DGE are proposed. They 


$$
\text { "chan" — 2008/7/31 — 11:51 — page } 80-\# 18
$$

are: experimentation-driven, experimental-theoretical-partnership and theorydriven. An empirical study of three participants exploring same Sketchpad task is described to illustrate these three approaches. The author understands that this proposal is based on result of only one empirical study. However, the finding in this study is interesting enough to suggest that this proposal may provide a fruitful direction to understand the development of utilization schemes ([13]) in DGE, viz. investigation of approaches of interplay between experimentation and theoretical considerations.

The followings are some possible directions for further research.

(1) What is the best way to describe the interplay between experimentation and theoretical consideration in the process of exploration in DGE?

(2) How does the approach of interplay relate to the problem-solver's background such as perception of DGE, perception of mathematics, experience of using dynamic geometry software and mathematics background?

(3) How does the approach of interplay relate to the specific mathematics task?

(4) How does the approach of interplay relate to the development of utilization schemes $([13])$ ?

(5) How does the approach of interplay relate to other theoretical perspectives such as variational dragging scheme $([7],[8],[9])$ ?

\section{Acknowledgement}

The author acknowledges the financial supports of HKU CRCG Conference Grants for Research Students and Sik Sik Yuen Education Research Fund. The empirical study reported in this paper is part of the author's $\mathrm{PhD}$ research study under the supervision of Dr Allen Leung.

\section{References}

[1] M. Artigue, Learning mathematics in a CAS environment: the genesis of a reflection about instrumentation and the dialectics between technical and conceptual work, International Journal of Computers for Mathematical Learning 7 (2002), 245-274.

[2] P. Drijvers and K. Gravemeijer, Computer algebra as an instrument: examples of algebraic schemes, in: The Didactical Challenge of Symbolic Calculators: Turning a Computational Device into Mathematical Instrument, (D. Guin, K. Ruthven and L. Trouche, eds.), Springer-Verlag, New York, 2005, 163-196. 


$$
\text { "chan" — 2008/7/31 — 11:51 — page } 81 \text { — \#19 }
$$

[3] E. P. Goldenberg and A. A. Cuoco, What is dynamic geometry?, in: Designing Learning Environments for Developing Understanding of Geometry and Space, (R. Lehrer and D. Chazan, eds.), Lawrence Erlbaum, Mahwah, 1998, 351-367.

[4] D. Guin and L. Trouche, Mastering by the teacher of the instrumental genesis in CAS environments: necessity of instrumental orchestrations, Zentralblatt für Didaktik der Mathematik 34 (2002), 204-211.

[5] D. Guin, K. Ruthven and L. Trouche (eds.), The Didactical Challenge of Symbolic calculators: Turning a Computational Device into a Mathematical Instrument, Springer Verlag, New York, 2005.

[6] B. Lins, Actual meanings, possible uses: secondary mathematics teachers and Cabri-Géomètre, paper presented in TG9 at the Third Annual Conference of European Research in Mathematics Education, 2003, http://www.dm.unipi.it/ didattica/CERME3/proceedings.

[7] A. Leung, Dynamic geometry and the theory of variation, in: Proceedings of PME 27: Psychology of Mathematics Education $27^{\text {th }}$ International Conference (Volume 3), (N. A. Pateman, B. J. Dougherty and J. T. Zilliox, eds.), University of Hawaii, Honolulu, 2003, 197-204.

[8] A. Leung and Y. C. Chan, Exploring necessary and sufficient conditions in dynamic geometry environments, International Journal for Technology in Mathematics Education 13 (2006), 37-43.

[9] A. Leung, Y. C. Chan and F. Lopez-Real, Instrumental genesis in dynamic geometry environments,, in: Proceedings of the ICMI 17 Study Conference (Part 2), (L. H. Son, N. Sinclair, J. B. Lagrange and C. Hoyles, eds.), Hanoi University of Technology, Vietnam, 2006, 346-353.

[10] A. S. Posamentier, Advanced Euclidean Geometry: Excursions for Secondary Teachers and Students, Key College Publishing, Emeryville, 2002.

[11] L. Trouche, Managing the complexity of human/machine interactions in computerized learning environments: guiding students' command process through instrumental orchestrations, International Journal of Computers for Mathematical Learning 9 (2004), 281-307.

[12] L. Trouche, An instrumental approach to mathematics learning in symbolic calculators environments, in: The Didactical Challenge of Symbolic Calculators: Turning a Computational Device into Mathematical Instrument, (D. Guin, K. Ruthven and L. Trouche, eds.), Springer-Verlag, New York, 2005, 137-162.

[13] P. Vérillon and P. Rabardel, Cognition and artifacts: a contribution to the study of thought in relation to instrumented activity, European Journal of Psychology of Education 10 (1995), 77-101.

YIP-CHEUNG CHAN

FACULTY OF EDUCATION

UNIVERSITY OF HONG KONG

POKFULAM ROAD

HONG KONG

E-mail: mathchan@graduate.hku.hk

(Received August, 2007) 